\title{
The OGLE-II event sc5_2859: a classical nova outburst?
}

C. Afonso 1,3 J. F. Glicenstein ${ }^{1}$, A. Gould ${ }^{2}$, M. C. Smith ${ }^{4}$, R. M. Wagner ${ }^{2}$, J. N. Albert ${ }^{5}$, J. Andersen ${ }^{8}$, R. Ansari ${ }^{5}$, É. Aubourg ${ }^{1}$, P. Bareyre ${ }^{1}$, J. P. Beaulieu ${ }^{6}$, G. Blanc ${ }^{1,12}$, X. Charlot ${ }^{1}$, C. Coutures $^{1,6}$, R. Ferlet $^{6}$, P. Fouqué ${ }^{9,10}$, B. Goldman ${ }^{1,3}$, D. Graff ${ }^{1}$, M. Gros ${ }^{1}$, J. Haissinski ${ }^{5}$, C. Hamadache ${ }^{1,13}$, J. de Kat ${ }^{1}$, L. LeGuillou ${ }^{1,11}$, É. Lesquoy ${ }^{1,6}$, C. Loup ${ }^{6}$, C. Magneville ${ }^{1}$, J. B. Marquette ${ }^{6}$, É. Maurice $^{7}$, A. Maury ${ }^{10}$, A. Milsztajn $^{1}$, M. Moniez ${ }^{5}$, N. Palanque-Delabrouille ${ }^{1}$, O. Perdereau ${ }^{5}$, L. Prévot ${ }^{7}$, Y. R. Rahal ${ }^{5}$, J. Rich ${ }^{1}$, M. Spiro ${ }^{1}$, P. Tisserand ${ }^{1}$, A. Vidal-Madjar ${ }^{6}$, L. Vigroux ${ }^{1,6}$, and S. Zylberajch ${ }^{1}$

${ }^{1}$ CEA, DSM, DAPNIA, Centre d'Études de Saclay, 91191 Gif-sur-Yvette Cedex, France e-mail: glicens@hep.saclay.cea.fr

2 Department of Astronomy, Ohio State University, Columbus, OH 43210, USA

3 Max-Planck fuer Astronomie, Koenigsthul 17, 69117 Heidelberg, Germany

${ }^{4}$ Kapteyn Astronomical Institute, University of Groningen, PO Box 800, 9700 AV Groningen, The Netherlands

5 Laboratoire de l'Accélérateur Linéaire, IN2P3 CNRS, Université de Paris-Sud, 91405 Orsay Cedex, France

${ }^{6}$ Institut d'Astrophysique de Paris, INSU CNRS, 98 bis Boulevard Arago, 75014 Paris, France

7 Observatoire de Marseille, 2 place Le Verrier, 13248 Marseille Cedex 04, France

8 Astronomical Observatory, Copenhagen University, Juliane Maries Vej 30, 2100 Copenhagen, Denmark

9 Observatoire Midi-Pyrénées, 14 Av. E. Belin, 31400 Toulouse, France

10 European Southern Observatory (ESO), Casilla 19001, Santiago 19, Chile

11 Instituut voor Sterrenkunde, Celestijnenlaan 200B, 3001 Leuven, Belgium

12 Osservatorio Astronomico di Padova, INAF, Vicolo dell'Osservatorio 5, 35122 Padova, Italy

13 Physique Corpusculaire et Cosmologie, Collège de France, 11 Pl. M. Berthelot, 75231 Paris Cedex 5, France

Received 3 August 2005 / Accepted 1 December 2005

\section{ABSTRACT}

Context. The OGLE-II event sc5_2859 was previously identified as the third longest microlensing event ever observed.

Aims. Additional photometric observations from the EROS (Expérience de Recherche d'Objets Sombres) survey and spectroscopic observations of the candidate star are used to test the microlensing hypothesis.

Methods. The combined OGLE and EROS data provide a high quality coverage of the light curve. The colour of the sc5_2859 event is seen to change with time. A spectrum taken in 2003 exhibits a strong $\mathrm{H}_{\alpha}$ emission line.

Results. The additionnal data show that the OGLE-II sc5_2859 event is actually a classical nova outburst.

Key words. stars: novae, cataclysmic variables - Galaxy: stellar contents - gravitational lensing

\section{Introduction}

Gravitational microlensing is now a well established tool for astrophysics. Of the more than 2000 microlensing events discovered to date, the great majority are in the direction of the Galactic bulge. The microlensing optical depth and the event timescale distribution obtained from these surveys provide unique tools for the study of Galactic structure and the mass functions of Galactic populations. Moreover, microlensing databases have proven to be an extremely rich source for the study of various classes of variable stars. In particular, they hold out the potential to obtain nova light curves with higher cadence and better precision than is usually the case.
The confusion between falling nova light curves and microlensing events is a potential problem for microlensing studies. The event sc5_2859, discussed in this paper, stands both as a concrete example of this confusion and as a warning about its impact on calculations of the optical depth. Novae have much larger outburst amplitudes than most microlensing events. But they are also easily distinguished from microlensing events from their very different light curves on the rising side of the peak. Hence, if these analyses excluded events with data points only over the descending part of the light curve, this potential confusion would have no practical effect on optical depth measurements. This is not the case, however, for two published optical-depth measurements (Alcock et al. 1997, 2000), which included, respectively, one and two decline-only events 
with long Einstein timescales $t_{\mathrm{E}}$. Specifically, event 124-A $\left(t_{\mathrm{E}}=76\right.$ days $)$, for the first paper and events 95-BLG-d11 ( $t_{\mathrm{E}}=62$ days) and 96-BLG-d14 ( $t_{\mathrm{E}}=47$ days) for the second. Here $t_{\mathrm{E}}$ is given by

$t_{\mathrm{E}}=\frac{\theta_{\mathrm{E}}}{\mu_{\mathrm{rel}}}, \quad \theta_{\mathrm{E}}=\sqrt{\kappa M \pi_{\mathrm{rel}}}$,

where $\theta_{\mathrm{E}}$ is the angular Einstein radius, $\pi_{\text {rel }}$ and $\mu_{\text {rel }}$ are the lens-source relative parallax and proper motion, $M$ is the lens mass, and $\kappa \equiv 4 G / c^{2} \mathrm{AU} \sim 8.1 \mathrm{mas} / M_{\odot}$. Although these events do not represent the bulk of the optical depth, they contribute significantly to it, since each event contributes $\propto t_{\mathrm{E}}$.

Three very long events with timescales greater than one year have been discovered in the OGLE-II and MACHO data sets: OGLE-1999-BUL-32/MACHO-99-BLG-22 (Mao et al. 2002; Bennett et al. 2002; Agol et al. 2002), OGLE-1999-BUL-19 (Smith et al. 2002) and BUL_SC5 244353/sc5_2859 (Smith 2003). The last of these, sc5_2859, was initially found by the OGLE-II collaboration and later identified by Smith (2003) as a long duration parallax event with one of the longest time scales ever observed, $t_{\mathrm{E}}=$ $547.6_{-2.8}^{+22.6}$ days, and as one of the first examples of disc-disc microlensing.

In this paper we present the light curve of the event sc5_2859 including additional points from the EROS 2 and OGLE projects databases. The new light curve and complementary spectroscopic observations of the candidate reveal that the event is most probably a nova outburst.

\section{Observational data}

The event sc5_2859 toward the Galactic bulge is a microlensing candidate from the OGLE-II catalog. The light curve in the $I$ band is publicly available ${ }^{1}$. The OGLE team did not observe the rising part of the light curve, whose peak occurred just before the 1997 bulge observation campaign. A detailed description of the telescope and instrument can be found in Udalski et al. (1997). The position of the source is RA $=17: 50: 36.09$ and Dec $=-30: 01: 46.6(l=359.6235, b=-1.4930)$. The location of the source is shown in Fig. 1.

After Smith (2003) presented a microlensing model of the event, which included the effects of microlensing parallax, the EROS 2 project searched its archival data for additional points that would allow a better constraint on the fit.

The observations of the EROS 2 data were carried out at La Silla, Chile with the $1 \mathrm{~m}$ MARLY telescope. The imaging was done simultaneously by two cameras (using a dichroic beam-splitter) composed of a mosaic of eight $2 \mathrm{~K} \times 2 \mathrm{~K}$ LORAL CCDs, with a field of view of $0.7(\alpha) \times 1.4(\delta)$ at an image scale of 0.' 6 per pixel. The EROS filters are non-standard: one camera observes in the $V_{\mathrm{E}}(420-720 \mathrm{~nm})$ filter and the other in the $I_{\mathrm{E}}$ filter $(620-920 \mathrm{~nm})$. The EROS and OGLE passbands are shown in Fig. 2. Note that both EROS $I_{\mathrm{E}}$ and $V_{\mathrm{E}}$ are sensitive to possible contributions from the $\mathrm{H} \alpha$ line. Approximate

\footnotetext{
1 The Web address for the OGLE catalog is http://www . astro.princeton. edu/ wozniak/dia/lens/
}

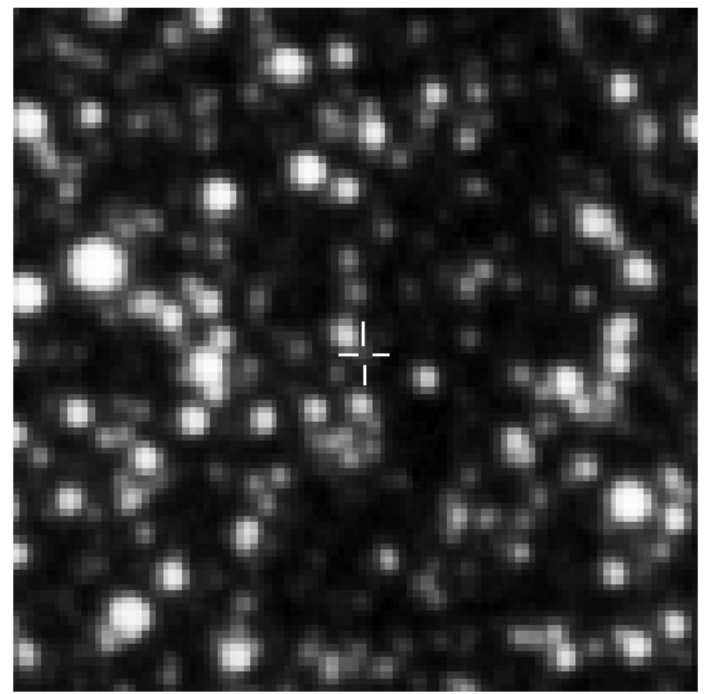

Fig. 1. EROS $I_{\mathrm{E}}$ image showing the location of the source of sc5_2859. The image size is $1^{\prime} \times 1^{\prime}$. The location of the source is indicated by the cross. North is up and east is to the left.

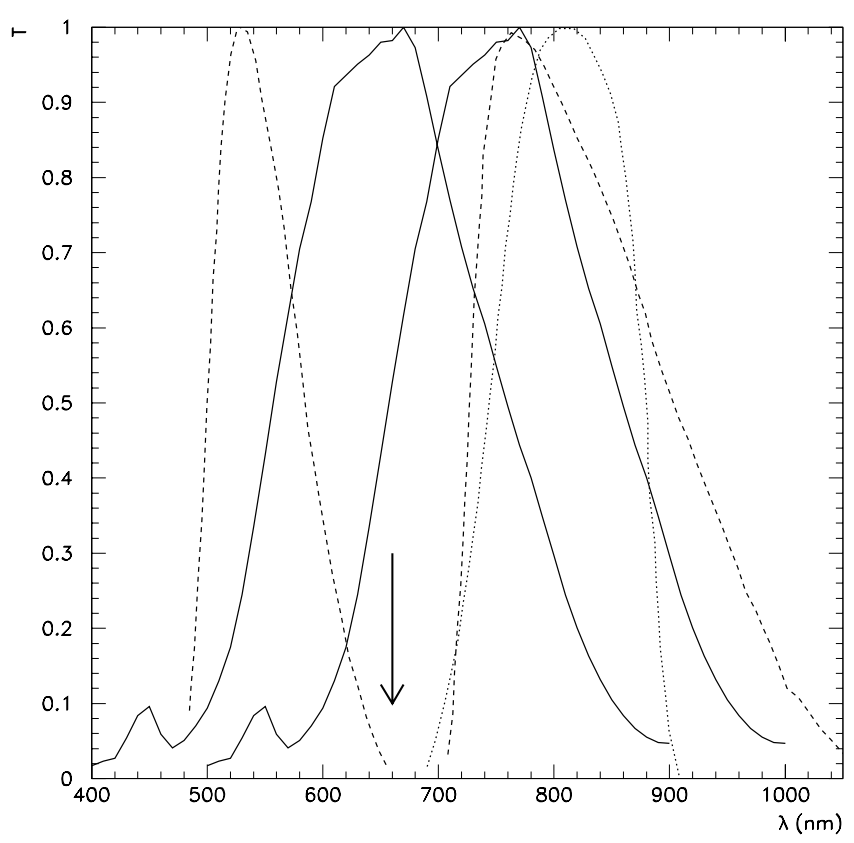

Fig. 2. EROS $V_{\mathrm{E}}$ and $I_{\mathrm{E}}$ passbands (solid lines). The broken lines show OGLE $V$ and $I$ passbands and the dotted line is Landolt $I$ passband. The arrow shows the position of the $\mathrm{H} \alpha$ line.

colour transformations from EROS filters to standard colours are given by:

$V_{\mathrm{E}}=0.69 V+0.31 I, \quad I_{\mathrm{E}}=-0.01 V+1.01 I$,

(Regnault 2000). However more precise colours were needed to compare the OGLE and EROS data sets. The colour transformations were found by comparing the approximate $V, I$ values of Eqs. (2) to the bulge $V, I$ maps of OGLE ${ }^{2}$. More information about the EROS instrument can be obtained from

\footnotetext{
2 These maps are available at web address

http://bulge.princeton.edu/ ogle/ogle2/bulge_maps.html
} 


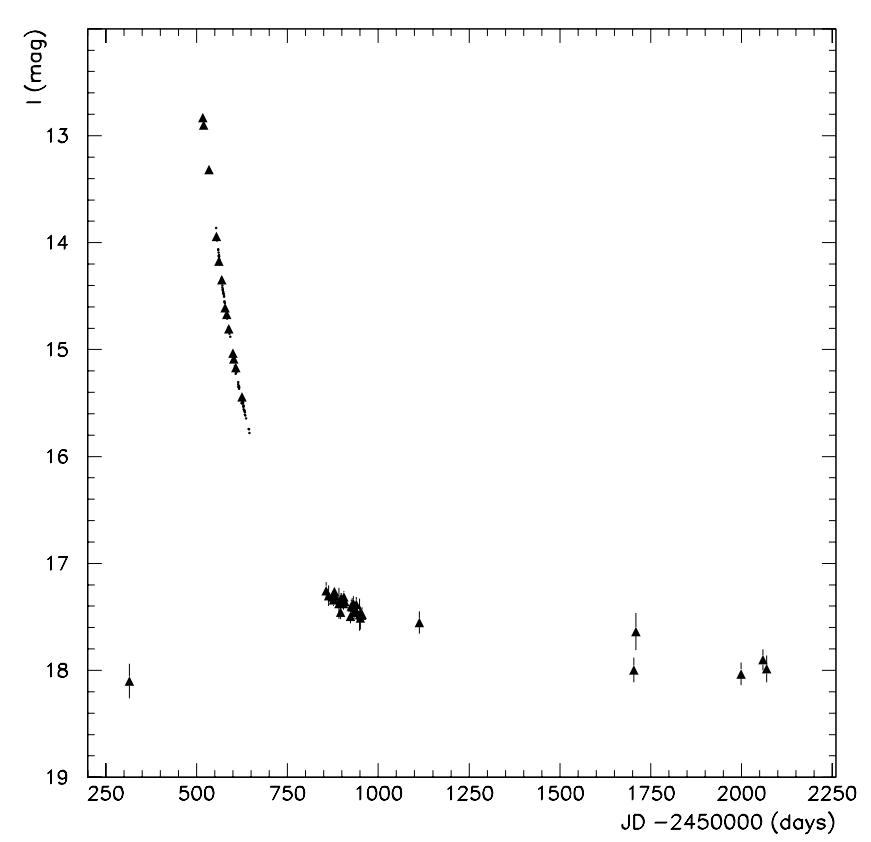

Fig. 3. Combined OGLE $I$ (dots) and EROS $I_{\mathrm{E}}$ (triangles) light curve for the OGLE sc5_2859 event. Note the data point close to the baseline flux value at $\mathrm{JD}=24500314.7$.

Bauer et al. (1997). The event sc5_2859 is located in the EROS field Cg001 close to the Galactic centre (see Afonso et al. 2003, for a Galactic plane map of the EROS 2 bulge fields).

\section{Light curve of sc5_2859}

The EROS data were processed by two different photometry pipelines. The PEIDA (Ansari 1996) PSF fitting photometry was run as part of the systematic photometry of EROS Galactic centre fields. The images were also processed with the ISIS (Alard 2000) differential photometry package. Differential photometry is more accurate and yields errors that are at the level of photon noise. In this paper, we mostly use the better differential photometry. Figure 3 shows the OGLE $I$ and EROS $I_{\mathrm{E}}$ light curves. The two data sets have been photometrically aligned by a linear transformation. The two data sets are in good overall agreement.

The most striking feature of the $I$ light curve (Fig. 3) is the EROS $I_{\mathrm{E}}$ data point at JD $=24500314.71$. The $I_{\mathrm{E}}$ flux at this date is at essentially the same level as it is 1700 days later, around JD $=2452100$. The $I_{\mathrm{E}}$ light curve rises from baseline to peak in less than 200 days, but requires more than 400 days to return to baseline. The strong asymmetry of the light curve is not easily explained by microlensing parallax. Unfortunately, no $V_{\mathrm{E}}$ pre-peak data are available. Although some images were taken, their quality is very poor due to bad weather conditions. Hence, the inference of strong asymmetry relies on a single $I_{\mathrm{E}}$ data point. Nevertheless, visual inspection of the image from which this datum was extracted reveals nothing anomalous.

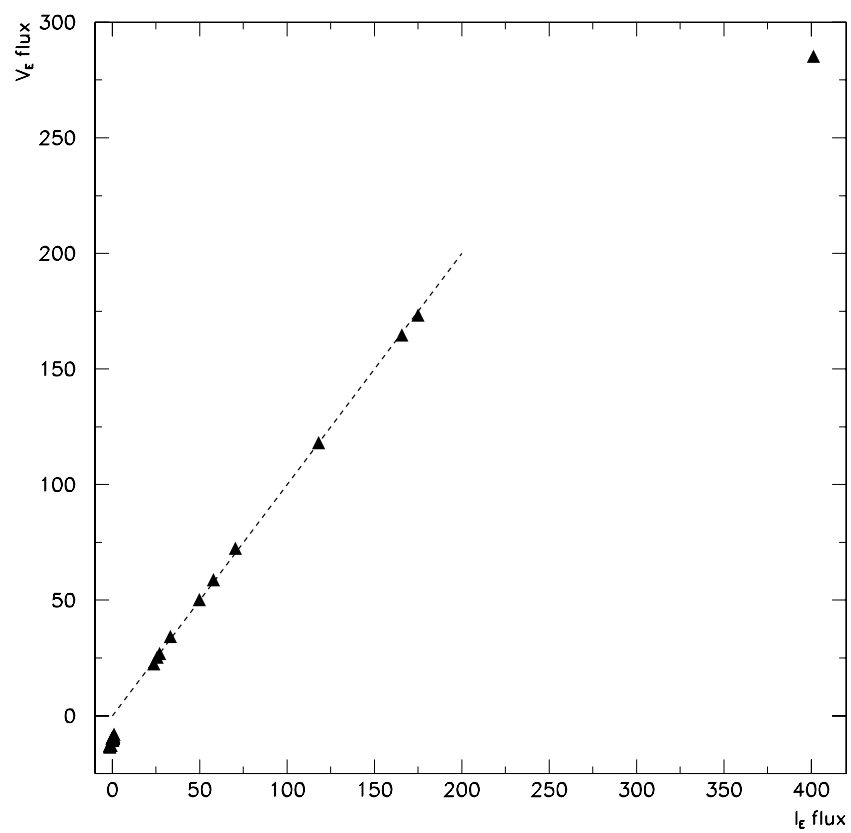

Fig. 4. Differential flux in the $V_{\mathrm{E}}$ band as a function of the differential flux in the $I_{\mathrm{E}}$ band. If sc5_2859 were a microlensing event, this curve would be a straight line, independently of the blending of the source. However, the curve deviates from a straight line (dotted line) at both small and large fluxes.

\section{Colour evolution sc5_2859}

Another critical piece of information provided by the EROS data is the evolution of the colour of sc5_2859 with time. EROS has obtained 41 colour measurements over more than 4 years. In addition, 11 more colour measurements were made available to us by the OGLE collaboration (Udalski 2005). The OGLE and EROS data are in apparent disagreement regarding the colour (see Figs. 5 and 6). The EROS passbands are wide and overlapping. The OGLE $V$ passband is close to Johnson $V$, while the $I$ passband is non-standard. We will show in Sect. 5.1 that the spectrum of the sc5_2859 object contains the $\mathrm{H} \alpha$ line in emission. This does not affect either OGLE passband, but affects both the EROS passbands. The usual colour transformation do not apply in this case. However, we will now show that both OGLE and EROS data show evidence for a chromaticity effect. This is sufficient to rule out the microlensing interpretation.

We now examine the colour changes from several different perspectives.

First, Fig. 4 shows the differential $V_{\mathrm{E}}$ flux versus differential $I_{\mathrm{E}}$ flux. The track of points shows strong curvature. By contrast, microlensing is achromatic and so (with a few exceptions to be noted below) predicts a strictly linear relation between fluxes in different bandpasses, independently of the blending of the source (see e.g. Fig. 4 of Afonso et al. 2001). The exceptions arise from cases in which the source colour varies spatially, so that during the course of the event the relative magnification of the blue and red source material varies. This can occur because of differential limb darkening when the lens transits the source (Witt 1995; Yoo et al. 2004) or because both 


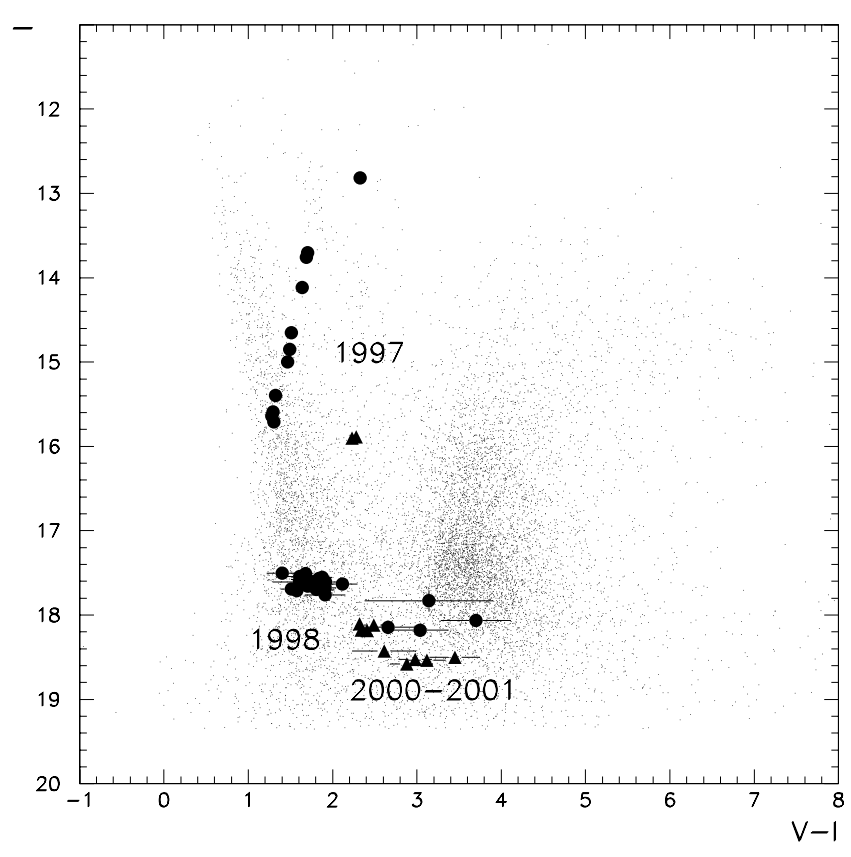

Fig. 5. Evolution of the sc5_2859 object in the local colour-magnitude diagram. The dots are EROS2 data points and the triangle are OGLE data points. The 1997 data can be interpreted as the end of the "constant bolometric luminosity" phase ("phase 2") and the 1998-2000-2001 data as the "white dwarf cooling phase".

components of a binary source are significantly magnified during the event, but by relative factors that vary with time. The first explanation is ruled out by the lack of finite-source effects in the light curve, which would always accompany significant colour changes. The second explanation is ruled out by the following argument. If the event is modeled as microlensing, then the peak observed flux corresponds to a magnification $A>250$ (and could be even higher if the source were heavily blended), corresponding to a projected lens-source separation (in units of $\theta_{\mathrm{E}}$ ) of $u \approx A^{-1}<0.004$, and hence a projected physical separation $b<0.03 \mathrm{AU}\left(\theta_{\mathrm{E}} / \mathrm{mas}\right)$. The putative binary source would have to have a projected separation $r_{\perp} \lesssim b$ if both sources were to contribute significantly to the light and yet leave the light curve without pronounced bumps. However, in this case, the orbital period would be $P \lesssim 1$ day $\left(\theta_{\mathrm{E}} / \mathrm{mas}\right)^{3 / 2}$ which would induce oscillatory (rather than the observed secular) colour changes on the light curve, unless $\theta_{\mathrm{E}}$ were extremely large, $\theta_{\mathrm{E}} \gtrsim 50$ mas. Such a large $\theta_{\mathrm{E}}$ would be implausible in its own right, but in addition (since $t_{\mathrm{E}} \sim 1$ year), this would imply $\mu_{\text {rel }} \gtrsim 50 \mathrm{mas} / \mathrm{yr}$, an impossibly large value. In brief, Fig. 4 essentially rules out the microlensing interpretation of this event.

Second, in Fig. 5, we show the evolution of the source colour and magnitude during the event superposed on a $[(V-I), I]$ colour-magnitude diagram (CMD) composed of field sources within a $5^{\prime} \times 5^{\prime}$ square centered on the event. EROS fluxes can be converted into standard $V, I$ magnitudes only at dates on which data in both passbands are available. The differential fluxes are first converted into absolute fluxes by adding a constant. This constant is found by fitting a linear transformation (with slope $\sim 1$ ) between the ISIS and the PEIDA fluxes. Finally, the fluxes are converted to standard magnitudes using the method described in Sect. 2. For most stars, this method gives a value close to the true standard V,I magnitudes. For the source of sc5_2859, the V, I magnitudes obtained are not the standard magnitude, as explained earlier in this section. The track of sc_2859 in the CMD is close to the true track for OGLE points, and it is basically the track that would be obtained in the EROS $\left[\left(V_{\mathrm{E}}-I_{\mathrm{E}}\right), I_{\mathrm{E}}\right] \mathrm{CMD}$, since the transformation from $\left(V_{\mathrm{E}}, I_{\mathrm{E}}\right)$ to $(V, I)$ is linear.

As mentioned above, microlensing events are in general achromatic, except for blending scenarios in which the source star is blended by one or more stars or in the presence of differential limb darkening. In the specific case of event sc5_2859, these exceptions are ruled out by the arguments presented at the beginning of this section. Thus the colour change in the CMD during the amplification of the light of the source star should depend monotonically on the magnitude. This is clearly contradicted by Fig. 5, where one can see (for EROS points) a blueward decline (points in 1997) from $(2.3,12.6)$ to $(1.4$, $15.5)$ and a clump at $(1.5,17.7)$ representing a slower redward decline (points in 1998), while the cluster of points just below the red clump are the baseline (points in 2000-2001). The OGLE points follow a qualitatively similar track in the CMD diagram.

The red giant clump is located at

$$
\left((V-I)_{\text {clump }}, I_{\text {clump }}\right) \simeq(3.8,17.4)
$$

in the local colour-magnitude diagram. Yoo et al. (2004) argue that the dereddened position of the red giant clump of the Galactic centre is located at

$[(V-I), I]_{0}^{\mathrm{GC}} \simeq(1.00,14.32)$.

The reddening and local extinction of the clump is thus

$\left[E(V-I), A_{I}\right]_{\text {clump }} \simeq(2.8,3.1)$,

which implies a ratio total-to-selective extinction $R_{V I}=$ $A_{V} / E(V-I)=2.1$ which is consistent with the values found by Sumi (2004a) for bulge fields.

Third, we show in Fig. 6 the time evolution of the $V-$ $I$ colour of the sc5_2859 object. The OGLE and EROS colours are obviously different. Figure 6 shows that the colour changes with time, first decreasing (for EROS data points) by $\sim 0.7 \mathrm{mag}$ between JD $=2450500$ and JD $=2450700$, then steadily increasing by 2 mag over the next 1400 days. The OGLE data points show a steady increase of the colour by $\simeq 0.7 \mathrm{mag}$ over 1200 days.

Apart from microlensing, the sc5_2859 event has a natural explanation as a cataclysmic variable $(\mathrm{CV})$ outburst. This interpretation, which was also suggested by Cieslinski et al. (2003), can be tested directly on the data and also by taking spectra of the candidate.

\section{Spectroscopic observations of the candidate}

\subsection{Observations}

Optical spectroscopy of the nova candidate was obtained with the Red Channel CCD spectrograph on the $6.5 \mathrm{~m} \mathrm{MMT}$ of 


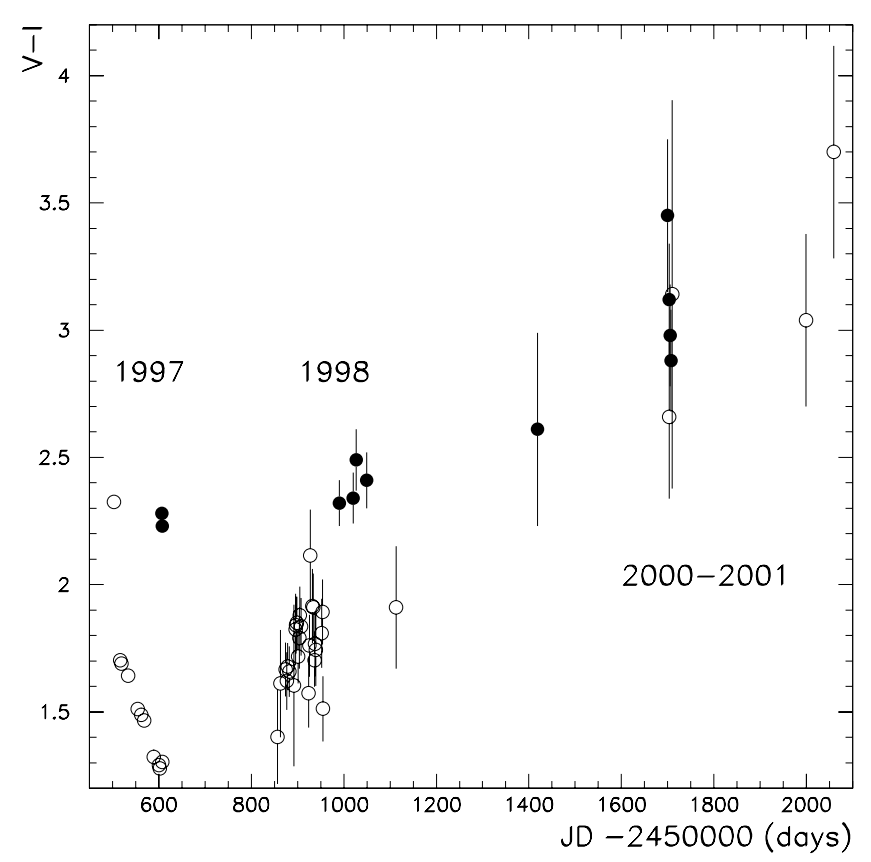

Fig. 6. Evolution of the $V-I$ colour of the sc5_2859 object with time. The open circle are EROS data points and the filled circle are OGLE data points. Evolution of the EROS colour: 1997: fades from $I=12.5$ to $I=15.7$. 1998: reddens from $V-I=1.4$ to $V-I=2.0 .2000-2001$ : near baseline at $V-I \sim 3$. The colour measured by OGLE increases, but more slowly between 1997 and 2000 .

the Smithsonian Institution and the University of Arizona on 2003 July 8.15 UT. Acquisition of the object on the 1 " wide entrance slit was accomplished by performing a blind offset from an anonymous nearby field star located at $\alpha=17: 50: 37.95$ and $\delta=-30: 01: 39.4$ (J2000), which was easily visible using the TV acquisition system (limiting magnitude $~ 21.5$ ). A 150 grooves $\mathrm{mm}^{-1}$ grating was employed that covered the spectral region 4000-9200 $\AA$ at a resolution of $19 \AA$. However, the signal-to-noise ratio $(\mathrm{S} / \mathrm{N})$ is poor below $5500 \AA$ and longward of $7500 \AA$. Two $1200 \mathrm{~s}$ exposures were obtained at starting airmasses of 2.16 and 2.27 respectively. The seeing averaged 1." 6 at these airmasses as measured by the FWHM of anonymous field stars on the slit. The transparency was of photometric quality based on spectrograph throughput measurements obtained earlier in the night. The spectrum of a HeArNe lamp was obtained immediately after the target spectra for accurate wavelength calibration. Three spectra were obtained near the end of the night of the standard star BD+28 4211 through a $5^{\prime \prime}$ wide entrance slit in order to correct the target spectra for instrumental response. Bias and flatfield spectra were obtained to facilitate the data reduction. The spectra were reduced, extracted, and calibrated using the IRAF software package.

\subsection{Results}

The extracted and calibrated spectrum of the nova candidate covering the 5500-7500 A region is shown in Fig. 7. The spectrum exhibits a strong and broad $\mathrm{H} \alpha$ emission line

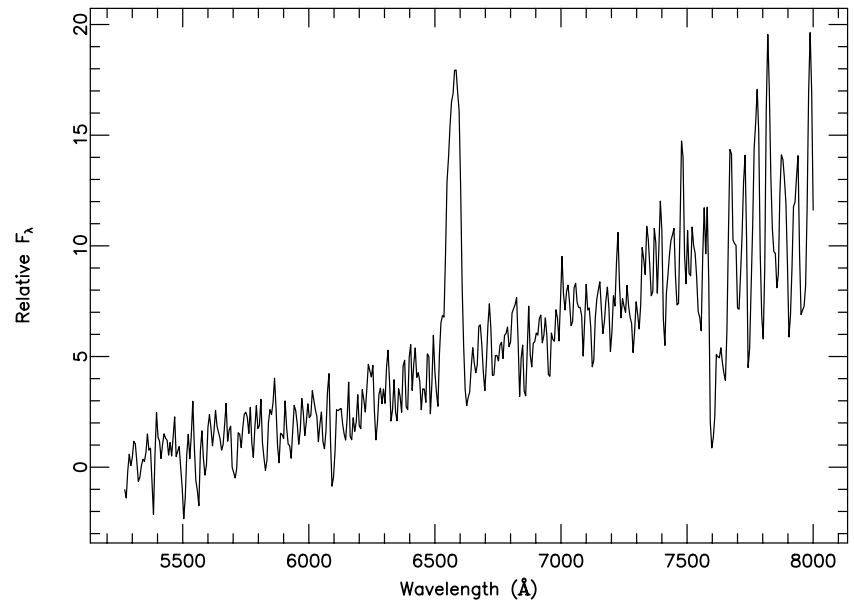

Fig. 7. Spectrum of the progenitor of the OGLE-II event SC5_2859 obtained with the $6.5 \mathrm{~m}$ MMT on 2003 July 8. The spectrum is dominated by a strong and broad $\mathrm{H} \alpha$ emission line superposed on a reddened continuum. The spectrum resembles that of some cataclysmic variables.

superposed on a reddened and noisy featureless continuum. The equivalent width of $\mathrm{H} \alpha$ emission is $176 \AA$ while its FWHM is $\simeq 2400 \mathrm{~km} \mathrm{~s}^{-1}$. The $\mathrm{S} / \mathrm{N}$ is too poor in the blue to confirm the presence of any other other emission lines such as $\mathrm{H} \beta$, He II $4686 \AA$, He I $5876 \AA$, and the C III-N III blend at $4650 \AA$. Similarly, strong interstellar absorption bands and absorption lines or bands of Na I D and $\mathrm{TiO}$ characteristic of a late-type companion star do not appear to be present, which is not surprising given our poor $\mathrm{S} / \mathrm{N}$. The data do not permit a significant limit to be placed on the reddening along the line of sight based on the strength of interstellar absorption bands.

\section{Discussion}

The spectrum of the stellar object associated the OGLE-II event SC5_2859 resembles a reddened cataclysmic variable (CV) star. These systems consist of a Roche-lobe filling main sequence star and a white dwarf accreting material through an accretion disc surrounding the compact object. The optical light is generally dominated by strong emission lines of the Balmer series of $\mathrm{H}, \mathrm{He}$, He II, and the $\mathrm{C} \mathrm{III} / \mathrm{N}$ III Bowen blend at 4640-4640 ̊ from the bright accretion disc. A comprehensive review of the properties of CVs is given by Warner (1995). In comparing this event with other CVs, the great strength of $\mathrm{H} \alpha$ emission and its breadth are both consistent with a system viewed at relatively high orbital inclination, close to $90^{\circ}$.

The presence of a CV associated with the outburst as measured by the OGLE and EROS experiments offers a natural explanation for the nature of the observed event. CVs are the progenitors of both dwarf novae and classical novae outbursts. The spectrum of this object is certainly consistent with other CVs that undergo dwarf nova outbursts such as U Gem and SS Cyg. The quiescent spectrum of these objects is dominated by strong Balmer emission lines accompanied by the lack of strong $\mathrm{He} \mathrm{I}$ and $\mathrm{He}$ II emission lines relative to $\mathrm{H} \beta$. In addition, 


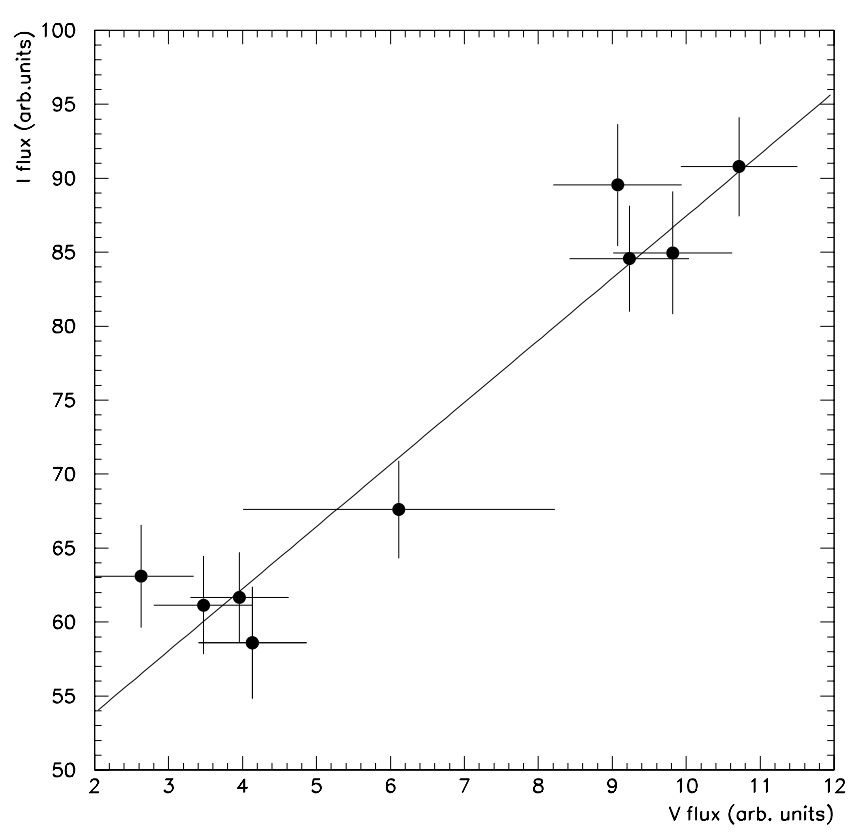

Fig. 8. $I_{\mathrm{OGLE}}$ flux versus $V_{\mathrm{OGLE}}$ flux during the "white dwarf cooling phase". The $I_{\mathrm{OGLE}}$ flux is a linear function of the $V_{\mathrm{OGLE}}$ flux because the $V-I$ colour of the dwarf is almost independent of temperature in the high temperature limit.

the $\mathrm{C}$ III/ $\mathrm{N}$ III blend is nearly always weak or absent in the quiescent spectra of dwarf novae. However, dwarf nova outbursts are relatively fast events with amplitudes of $\simeq 2-4$ mag and durations of less than $\sim 20$ days. In addition, they show a recurrence time scale $\sim 100$ days or less. The light curve of OGLE SC5_2859 is inconsistent with the dwarf nova interpretation since the duration of the outburst is considerably longer and it is not observed to recur over the $\sim 1300$ days of monitoring.

The most likely interpretation of the sc5_2859 event is that it is a classical nova outburst. Classical novae occur in CV binary systems as a result of a thermonuclear runaway on the surface of the white dwarf primary star. The time evolution of classical novae outbursts can be separated into 4 phases. These phases are 1) the rise to maximum visual light; 2) a period of constant bolometric luminosity; 3) a period of cooling of the white dwarf; and 4) the re-establishment of accretion from the secondary. The evolutionary track of classical novae remnants is shown in Fig. 5.1 of Bode \& Evans (1989). This figure uses nova DQ Her 1934, for which the transition between phases 2 and 3 occurs at $M_{V} \simeq 7$. The $V$ magnitude changes by more than 10 units during phase 2 . The track of the sc5_2859 object in the local $(V-I, I)$ CMD is displayed in Fig. 5. The 1997 data may be identified with the end of the "constant bolometric luminosity" period and the 1998-2000-2001 data with the "white dwarf cooling phase". The white dwarf cooling phase thus occurs after the turning point in the colour evolution around $\mathrm{JD}=2450860$ days.

We now find the distance of the nova from the evolution of colour versus time. For this, we use only OGLE colours, which are more reliable, since they are not affected by the $\mathrm{H} \alpha$ line. Figure 8 shows a plot of $I_{\text {OGLE }}$ flux versus $V_{\text {OGLE }}$ flux during the "white dwarf cooling phase". This plot is very well fitted by a straight line with slope $s=4.2 \pm 0.4$. A possible interpretation is as follows: the flux comes from the blend of two objects: one with $I$ and $V$ fluxes that are constant in time and another with $I$ and $V$ fluxes decreasing with time, but with constant $V-I$ colour. Translating the value of the slope $s$ in term of $V-I$ colour gives: $(V-I)^{\mathrm{WD}}=1.56$.

A high-temperature white dwarf cooling is expected to have an almost constant $V-I$ colour. This is because the spectrum of a high-temperature white dwarf cooling changes mainly in the UV. This qualitative argument is supported by the calculations of Bergeron et al. (1995). They find that the $V-I$ colour of a hydrogen-atmosphere white dwarf with $\log g=8$ and temperature $>30000 \mathrm{~K}$ should be within 0.05 mags of the asymptotic value $(V-I)^{H}=-0.36$ (see also their Fig. 6). Using the theoretical value $(V-I)^{H}=-0.36$, one finds for sc5_2859, $E(V-I)=1.56-(-0.36)=1.92$. If a helium atmosphere model is used instead, one gets $E(V-I)=1.82$. These correspond respectively to $68.5 \%$ and $65 \%$ of the dust column to the Galactic centre.

The projected height of the field is $4.0 \mathrm{deg} \times 8 \mathrm{kpc}=$ $560 \mathrm{pc}$, while the scale height of dust is $130 \mathrm{pc}$. Hence, for the nova to lie in front of $68.5 \%$ the dust, a hydrogen atmosphere white dwarf would have to be at a height of $z=$ $-130 \mathrm{pc} \ln 0.315=150.2 \mathrm{pc}$ and so at a distance of $D^{\mathrm{WD}}=$ $8 \mathrm{kpc}(150.2 \mathrm{pc} / 560 \mathrm{pc})=2140 \mathrm{pc}$. A similar calculation gives a distance of $1950 \mathrm{pc}$ for a helium atmosphere dwarf. Taking the average value as the distance, we find

$D^{\mathrm{WD}}=2045 \pm 95 \pm 500 \mathrm{pc}$,

where the first error represents the uncertainty for the $\mathrm{H} / \mathrm{He}$ ambiguity of the WD composition and the second error represents our rough estimate in the uncertainty in estimating the distance from the relative extinction.

Around JD $=2451704$, the apparent magnitude of the sc5_2859 object is $V=21.5 \pm 0.05$. Recalling that the exinction to the nova is $A_{V}=R_{V I} E(V-I)=4$, the absolute $V$ magnitude of the source (assuming it is a hydrogen atmosphere WD) is then

$M_{V}(\mathrm{JD}=2451704) \simeq 5.8$.

This is compatible with the minimum $M_{V}$ values given in Table 6 of Downes \& Duerbeck (2000). However, the $M_{V}$ value of sc_2859 might be somewhat lower than 5.8, since it is very likely that the source is blended. Note that the OGLE collaboration found that the source of sc5_2859 had a very high apparent proper motion which they interpreted as the effect of a blend (Sumi 2004b).

Since the earliest EROS $V_{\mathrm{E}}$ point has $V \simeq 15$, it is likely that most of the early decline part of the light curve (interpreted as "phase 2") was missed. The speed class of the nova can only be guessed at by means of qualitative arguments. The "transition part" of the V light curve is smooth and steadily declining. This favours a fast or very fast nova. Other features give only weak constraints. The slope of the $V$ light curve around $\mathrm{JD}=2450600$ is

$\frac{\mathrm{d} V}{\mathrm{~d} t} \simeq 0.02 \mathrm{mag} / \mathrm{day}$ 
but this is only a lower limit to the early-decline slope. A similar constraint comes from the date of the first data point of the $I_{\mathrm{E}}$ light curve, which is observed before the outburst. These arguments favour a fast nova outburst.

Another useful check of the nova hypothesis is the calculation of the $\mathrm{H} \alpha$ line flux. The ratio of the $R$ band continuum flux to the $I$ band continuum flux can be estimated from the spectrum. Transforming to magnitudes using Table 2.1 of Binney \& Merrifield (1998), one finds that

$R^{\mathrm{cont}}-I=1.53$

around JD $=2452829$, so that $R \simeq 20$. The $R$ absorption is $A_{R} \simeq 3$. The $R^{\text {cont }}$ value corresponds to a continuum flux in the $R$ band of $2.3 \times 10^{32} \mathrm{erg} / \mathrm{s}$ if the sc5_2859 object is located at $2.05 \mathrm{kpc}$. The ratio of the $\mathrm{H} \alpha$ line flux to the continuum $R$ band flux is roughly 0.5 , so the $\mathrm{H} \alpha$ line flux is roughly $10^{32} \mathrm{erg} / \mathrm{s}$. $\mathrm{H} \alpha$ line fluxes of respectively 1,3 and $4 \times 10^{32} \mathrm{erg} / \mathrm{s}$ are expected for very fast, fast and moderately fast, 6.5 year old classical nova outbursts, according to Downes et al. (2001). The observed $\mathrm{H} \alpha$ line flux is thus in agreement with the hypothesis of a fast or very fast nova.

The paragraphs above summarize the arguments in favour of the classical nova hypothesis. It is also possible in principle that the sc5_2859 event is related to the subclass of recurrent novae such as T CrB, RS Oph, V3890 Sgr, and V745 Sco. The lack of obvious spectral features arising from a late-type giant star in the red weakens this association, however, in spite of the similarity of the light curve to some other recurrent novae. Nevertheless, a search for previous outbursts in archival plate material might prove worthwhile.

Acknowledgements. We thank A. Udalski for interesting comments and for making unpublished OGLE data on sc5_2859i available to us. We are grateful to D. Lacroix and the technical staff at the Observatoire de Haute Provence and A. Baranne for their help in refurbishing the MARLY telescope and remounting it in La Silla. We are also grateful to the technical staff of ESO, La Silla for the support given to the EROS project. We thank J.-F. Lecointe and A. Gomes for the assistance with the online computing. Work by A. Gould was supported by NSF grant AST 042758. M.C. Smith acknowledges financial support from The Netherlands Organisation for Scientific Research.

\section{References}

Afonso, C., Albert, J. N., Andersen, J., et al. (EROS collaboration) 2001, A\&A, 378, 1014

Afonso, C., Albert, J. N., Alard, C., et al. (EROS collaboration) 2003, A\&A, 404, 145

Agol, E., Kamionkowski, M., Koopmans, L. V. E., \& Blanford, R. D. 2002, ApJ, 576, L131

Alard, C. 2000, A\&AS, 144, 363

Alcock, C., Allsman, R. A., Alves, D., et al. (MACHO collaboration) 1997, ApJ, 479, 119

Alcock, C., Allsman, R. A., Alves, D. R., et al. (MACHO collaboration) 2000, ApJ, 541, 734

Ansari, R. 1996, Vistas in Astronomy, 40, 519

Bauer, F., et al. (EROS collaboration) 1997, Proc. of the "Optical Detectors for Astronomy" workshop, ESO

Bennett, D. P., Becker, A. C., Quinn, J. L., et al. 2002, ApJ, 579, 639

Bergeron, P., Wesemael, F., \& Beauchamp, A. 1995, PASP, 107, 1047

Binney, J., \& Merrifield, M. 1998, Galactic Astronomy (Princeton: Princeton University Press)

Bode, M. F., \& Evans, A. 1989, Classical novae (New York: Wiley)

Cieslinski, D., Diaz, M. P., Mennickent, R. E., \& Pietrzynski, G. 2003, PASP, 115,193

Downes, R. A., \& Duerbeck, H. W. 2000, AJ, 120, 2007

Downes, R. A., Duerbeck, H. W., \& Delahodde, C. E. 2001, J. Astron. Data, 7, 6

Mao, S., Smith, M. C., Wozniak, P., et al. 2002, MNRAS, 329, 349

Popowski, P., Cook, K. H., Drake, A. J., et al. (MACHO collaboration) 2000, in Microlensing 2000, ed. J. W. Menzias, \& P. D. Sackett, ASP Conf. Ser.

Regnault, N. 2000, Ph.D. Thesis, Université de Paris 7, LAL 00-65

Reid, N. 1991, AJ, 102, 1428

Smith, M. C. 2003, MNRAS. 343, 1172

Smith, M. C., Mao, S., \& Wozniak, P. 2002, MNRAS, 336, 670

Sumi, T. 2004a, MNRAS, 349, 193

Sumi, T. 2004b, MNRAS, 348, 1439

Sumi, T., Abe, F., Bond, I. A., et al. 2003, ApJ, 591, 204

Udalski, A. 2005, private communication

Udalski, A., Szymanski, M., Stanek, K. Z., et al. 1994, Act. Astr., 44, 165

Udalski, A., Kubiak, M., \& Szymanski, M. 1997, Acta Astr., 47, 319

Udalski, A., Zebrun, K., Szymanski, M., et al. 2000, Act. Astr., 50, 1

Warner, B. 1995, Cataclysmic Variable Stars (Cambridge: Cambridge University Press)

Witt, H. J. 1995, ApJ, 449, 42

Yoo, J., DePoy, D. L., Gal-Yam, A., et al. 2004, ApJ. 603, 139 